\title{
Fuerte evidencia de que la actividad física disminuye el dolor y mejora la funcionalidad en pacientes con artrosis del miembro inferior
}

Strong evidence for exercise to reduce pain and to improve function in patients with lower limbosteoarthritis.

\section{Objetivos}

Evaluar la eficacia de la actividad física para el tratamiento del dolor y la mejora de la funcionalidad en las personas con artrosis de miembro inferior, ya sea de rodilla o de cadera; comparando las distintas formas de actividad física entre sí o bien con un grupo control que no realizara ninguna actividad.

\section{Fuentes de datos}

La búsqueda se realizó en Medline, Embase, CINAHL, AMED, HMIC (Health Management Information Consortium), Cochrane Database of Systematic Reviews Cochrane Controlled Clinical Trials (CENTRAL), DARE (Database of Reviews of Effectiveness), NHS EED (Economic Evaluations Database), y Web of Science; hasta marzo de 2012.

\section{Selección de estudios}

Se incluyeron ensayos clínicos aleatorizados de pacientes adultos con diagnóstico clínico o radiológico de artrosis de cadera o rodilla. La intervención debía ser cualquier tipo de ejercicio terapéutico sin importar el contenido, la duración, la frecuencia o la intensidad. Como comparadores se tomaron otras formas de ejercicio o un grupo control que no realizara ninguna actividad.

Las medidas de resultado debían ser al menos una medida reportada de dolor y la función física evaluada con la escala de
Kuyken W y col. The Lancet 2015;386(9988):63-73. discapacidad Western Ontario and McMaster Universities (WOMAC).

\section{Extracción de datos}

Fue realizada por seis revisores que trabajaron de a pares y en forma independiente, tanto para la obtención de los datos de eficacia como para la evaluación de calidad de los estudios. Dos investigadores clasificaron independientemente los ejercicios de la intervención en tres categorías definidas a priori, según estuviesen destinados a mejorar la fuerza, la flexibilidad o la capacidad aeróbica y la salud general.

\section{Resultados principales}

El análisis secuencial de los 60 estudios incluidos, con un total de 8.218 pacientes, reportó beneficios de cualquier tipo de ejercicio físico comparado con el sedentarismo o la falta de actividad física, tanto para el dolor como para la funcionalidad física del miembro inferior con artrosis. Las intervenciones combinadas de fortalecimiento, flexibilidad y ejercicio aeróbico parecerían más beneficiosas en la mejora de la funcionalidad y el dolor del miembro inferior (ver tabla 1).

A pesar de ello, los estudios evaluados no tuvieron suficiente poder estadístico para concluir en los beneficios de un ejercicio por sobre otro, según su variedad, frecuencia, intensidad o duración adecuada.

Tabla 1. Principales resultados del metanálisis comparando los distintos tipos de intervenciones físicas frente a ninguna actividad, en la mejora de la funcionalidad y el dolor del miembro inferior (rodilla, cadera o varias articulaciones) con ajuste de variables para un año de seguimiento.

\begin{tabular}{|c|c|c|c|}
\hline \multirow[t]{2}{*}{ Resultado } & \multirow[t]{2}{*}{ Tipo de actividad física } & Sin ajuste de variables & Con ajuste de variables \\
\hline & & DME (IC 95\%) & DME (IC 95\%) \\
\hline \multirow[t]{4}{*}{ Funcionalidad } & Flexibilidad & $-0,17(-1,26$ a 0,93$)$ & $-0,14(-1,26$ a 0,97$)$ \\
\hline & Fortalecimiento & $-0,37(-0,84$ a 0,09$)$ & $-0,36(-0,87$ a 0,14$)$ \\
\hline & Ejercicio aeróbico & $-0,30(-1,53$ a 0,92$)$ & $-0,46(-1,71$ a 0,80$)$ \\
\hline & Resultado combinado & $-0,63(-1,16$ a $-0,10)$ & $-0,57(-1,17$ a 0,03$)$ \\
\hline \multirow{4}{*}{ Dolor } & Flexibilidad & $-0,66(-1,33 \mathrm{a} 0)$ & $-0,62(-1,31$ a 0,07$)$ \\
\hline & Fortalecimiento & $-0,81(-1,13 a-0,50)$ & $-0,74(-1,09 a-0,39)$ \\
\hline & Ejercicio aeróbico & $-0,41(-1,13$ a 0,30$)$ & $-0,33(-1,08$ a 0,40$)$ \\
\hline & Resultado combinado & $-0,69(-1,04$ a $-0,35)$ & $-0,64(-1,04 a-0,26)$ \\
\hline
\end{tabular}

$\mathrm{DME}=$ diferencia de medias estandarizada

\section{Conclusiones}

Esta revisión sistemática y metanálisis reporta ciertos beneficios de la actividad física comparada con el sedentarismo, como la mejoría de la funcionalidad y del dolor, en los individuos con osteoartrosis. Sin embargo no fue posible determinar la superioridad de un tipo de ejercicio sobre otro, ni la frecuencia o la intensidad óptimas.
Fuente de financiamiento: No referida en el artículo. Los autores, a partir de una comunicación personal, informaron que la financiación de este trabajo se encuentra dentro del marco de un programa de beca de 5 años para el estudio del manejo de la artrosis en atención primaria. 


\section{Comentario}

La artrosis es una enfermedad crónica y progresiva, cuyo tratamiento sintomático y paliativo debe ser bien enfocado. Existe evidencia radiológica en más del $50 \%$ de las personas mayores de 50 años y en el $80 \%$ de mayores de 75 años de signos de artrosis (principalmente de miembros inferiores, cadera o rodilla) ${ }^{1}$. Se sabe que los principales factores de riesgo para desarrollar este problema son la edad, el sexo femenino y el sobrepeso ${ }^{2,3,4,5}$. Considerando que la expectativa de vida es cada año más larga y la epidemia de obesidad es mundial, podemos proyectar un aumento sustancial de la prevalencia de artrosis en un futuro cercano. Por todo lo expuesto, es sumamente importante el correcto abordaje de la patología en atención primaria de la salud. Los resultados de esta revisión muestran de manera concluyente que con una mínima actividad física ya se observan mejorías significativas en el dolor y la fun- ción física de los pacientes con artrosis.

Investigaciones futuras deberían tener como objetivo comparar los beneficios de los distintos tipos de actividad física, con variantes de frecuencia, duración e intensidad, evitando comparar esta intervención contra el sedentarismo o la falta de actividad física.

\section{Conclusiones del comentador}

Los resultados obtenidos por este metanálisis demuestran los beneficios y la importancia de la incorporación de la actividad física en el tratamiento de la artrosis de miembro inferior, la que debería estar indicada como parte del tratamiento de los pacientes con este problema de salud.

Denise Cytryn [ Médica especialista en Medicina Familiar, Servicio de Medicina Familiar y Comunitaria, Hospital Italiano de Buenos Aires. denise.cytryn@ hospitalitaliano.org.ar ]

Cytrin D. Fuerte evidencia de que la actividad física disminuye el dolor y mejora la funcionalidad en pacientes con artrosis del miembro inferior. Evid Act Pract Ambul. 2016 ;19(1)8-9. Ene-Mar. Comentado de: Uthman OA, y col. Exercise for lower limb osteoarthritis: systematic review incorporating trial sequential analysis and network meta-analysis. BMJ. 2013;347(f5555). PMID: 24055922.

\section{Referencias}

1. Frusso R. Osteoartritis. En:Medicina Familiar y Práctica Ambulatoria 2da Ed. Editorial Médica Panamericana, 2006, p 1680-4.

3. Jordan JM, y col. Self-reported functional status in osteoarthritis of the knee in a rural southern community: the role of sociodemographic factors, obesity, and knee pain. Arthritis Care Res. 1996;9(4):273.

3. Felson DT, y col. Weight loss reduces the risk for symptomatic knee osteoarthritis in women. The Framingham Study. Ann Intern Med. 1992;116(7):535.

4. Hartz AJ, y col. The association of obesity with joint pain and osteoarthritis in the HANES data. J Chronic Dis. 1986;39(4):311.

5. Davis MA, y col. Sex differences in osteoarthritis of the knee. The role of obesity. Am J Epidemiol. 1988;127(5):1019.

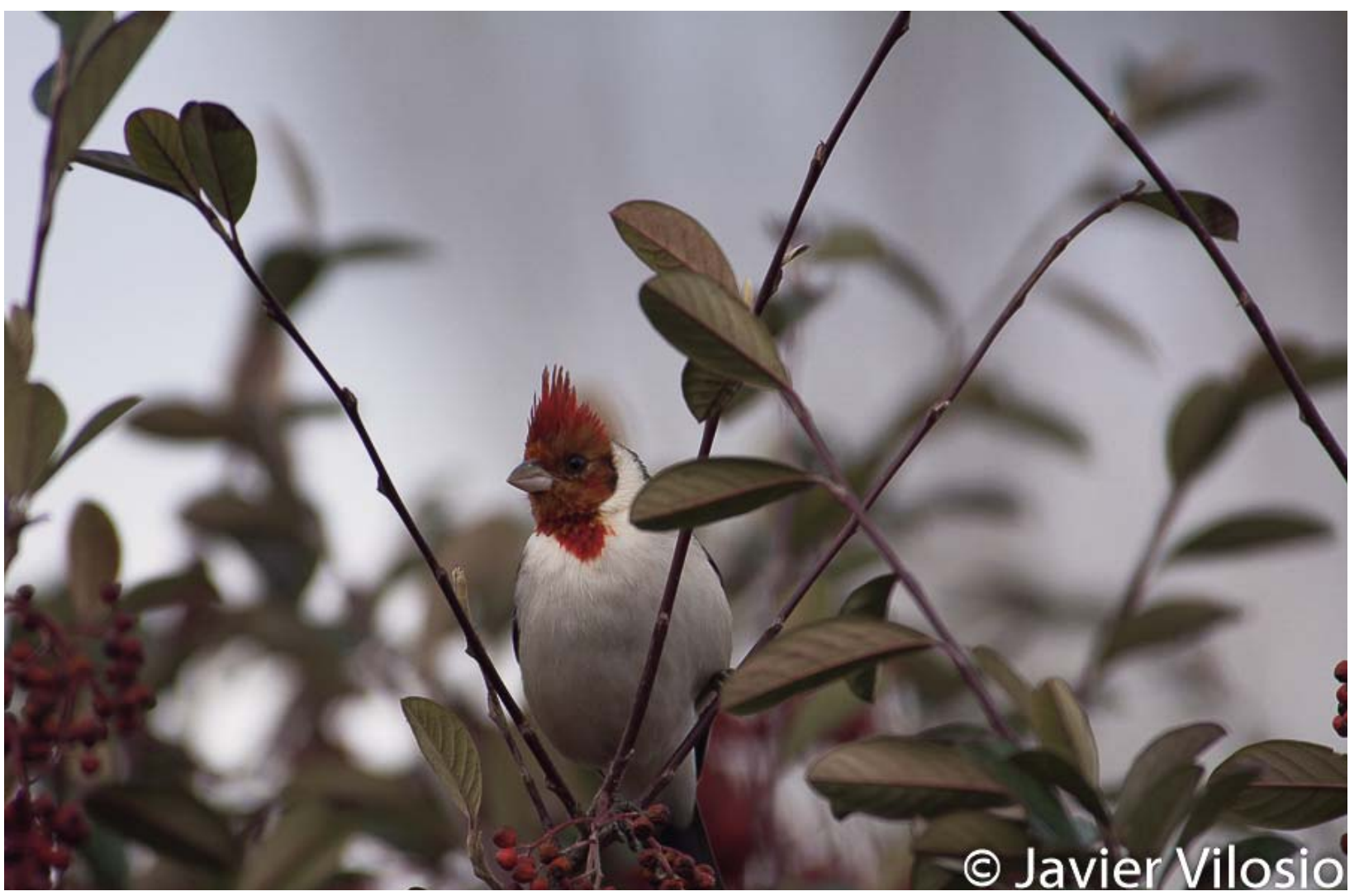

Fotografía: Javier Vilosio 